01

\title{
Решение задачи о форме вертикального жидкого моста между выпуклыми поверхностями с учетом силы тяжести
}

\author{
(c) Е.В. Галактионов, ${ }^{1}$ Н.Е. Галактионова, ${ }^{2}$ Э.А. Тропп ${ }^{1}$ \\ ${ }^{1}$ Физико-технический институт им. А.Ф. Иофрфе РАН, \\ 194021 Санкт-Петербург, Россия \\ ${ }^{2}$ Санкт-Петербургский политехнический университет Петра Великого, \\ 195251 Санкт-Петербург, Россия \\ e-mail: evgalakt@mail.ru
}

Поступило в Редакцию 20 мая 2020 г.

В окончательной редакции 27 сентября 2020 г.

Принята к публикации 29 сентября 2020 г.

\begin{abstract}
Представлено решение задачи о форме боковой поверхности вертикального трехмерного катеноидального жидкого моста малого объема между двумя произвольными выпуклыми твердыми поверхностями в осесимметричном случае с учетом действия силы тяжести. Дана вариационная постановка исходной задачи. Решение найдено методом итераций в предположении малости числа Бонда. Предложен алгоритм итерационного процесса. Обнаружены области изменения параметров, для которых отсутствует единственность решения задачи. Установлено, что максимальное число различных профилей боковой поверхности жидкого моста, соответствующих одному выбранному набору параметров, равно четырем. В качестве примера решена задача о форме жидкого моста между двумя сферами.
\end{abstract}

Ключевые слова: форма жидкого моста, метод итераций, малый параметр.

DOI: 10.21883/JTF.2021.03.50513.173-20

\section{Введение}

В наших работах [1,2] была решена задача о форме вертикального катеноидального жидкого моста между двумя твердыми горизонтальными плоскостями. Такая задача возникает, в частности, при изучении формы жидких менисков, образующихся в процессе роста кристаллов по способу Степанова [3]. Однако, как известно, фронт кристаллизации в этом процессе в действительности не является плоским, а представляет собой выпуклую поверхность малой кривизны. Вследствие этого задача о нахождении формы жидкого моста между двумя произвольными выпуклыми твердыми поверхностями является актуальной. Для решения задач такого типа используются как численные, так и асимптотические методы. В работе [4] построена асимптотика формы поверхности горизонтального жидкого моста между двумя вертикальными твердыми плоскостями при малых числах Бонда. Оригинальный подход к нахождению формы жидкого моста между сферами, основанный на решении обратной задачи, используется в $[5,6]$. В первой из этих работ дается классификация форм мостов, а во второй приводятся их фотографии, полученные в эксперименте. Подробная библиография работ, посвященных решению задач о форме жидких мостов и возможным приложениям этих исследований, приведена в [7].

В настоящей работе дана вариационная постановка задачи о форме боковой поверхности вертикального трехмерного катеноидального жидкого моста малого объема, находящегося между двумя произвольными выпуклыми твердыми поверхностями. Рассмотрен осесимметричный случай, и для решения задачи используются цилиндрические координаты. Учитывается действие силы тяжести. Предполагается, что число Бонда есть малый параметр задачи. Предложен алгоритм итерационного процесса решения задачи. В качестве примера построено решение задачи о форме жидкого моста между сферами.

\section{1. Вертикальный жидкий мост. Вариационная постановка задачи}

Рассмотрим случай вертикального жидкого моста, находящегося между двумя твердыми выпуклыми поверхностями (дном и крышкой) (рис. 1). Ввиду предполагаемой осевой симметрии будем решать задачу о нахождении профиля боковой поверхности жидкого моста в цилиндрической системе координат $(r, z)$. Поверхностные натяжения между средами: $\alpha_{13}, \alpha_{14}, \alpha_{34}, \alpha_{23}$, $\alpha_{24}$ соответственно. Область контакта жидкого моста с поверхностью $z=f_{1}(r),\left(f_{1}(0)=0\right)$ (дном) - круг радиуса $r_{1}$, а с поверхностью $z=\hat{f}_{2}(r)=\hat{h}+f_{2}(r)$, $\left(f_{2}(0)=0\right)$ (крышкой $)-$ круг радиуса $r_{2}$. Обозначим $u_{1}(r), u_{2}(r)$ - искомые функции, описывающие профили нижней $\left(u_{1}(r)\right)$ и верхней $\left(u_{2}(r)\right)$ частей боковой поверхности жидкого моста. Область, разделяющая эти части („шейка“) - круг радиуса $r_{*}\left(r_{*} \geq 0\right)$. В настоящей работе будем рассматривать жидкий мост, имеющий катеноидальную форму [8]: $r_{*}<\min \left\{r_{1}, r_{2}\right\}, u_{1}^{\prime}(r)<0$, $r \in\left[r_{*}, r_{1}\right], u_{2}^{\prime}(r)>0, r \in\left[r_{*}, r_{2}\right]$. Предположим дополнительно, что углы $\theta_{1}, \theta_{2}$ удовлетворяют следующим условиям: $0<\theta_{i}<\pi / 2-\arctan \left|f_{i}^{\prime}\left(r_{i}\right)\right|, i=1,2$, 
т.е. имеет место смачивание жидкостью твердых поверхностей [3]. Так как нет никаких физических причин для появления заострения профиля боковой поверхности моста, то в точке с абсциссой $r=r_{*}$ касательная к профилю должна быть вертикальной: $u_{1}^{\prime}\left(r_{*}\right)=-\infty, u_{2}^{\prime}\left(r_{*}\right)=+\infty, u_{1}\left(r_{*}\right)=u_{2}\left(r_{*}\right)$. Заметим, что в силу приведенных выше ограничений функции $u_{1}(r)$ и $u_{2}(r)$ будут однозначными функциями.

Объем жидкого моста считаем фиксированным:

$$
\begin{aligned}
& I\left\{u_{1}(r), u_{2}(r)\right\}=2 \pi\left(\int_{r_{*}}^{r_{1}}\left(u_{1}(r)-f_{1}(r)\right) r d r\right. \\
& \left.+\int_{r_{*}}^{r_{2}}\left(\hat{f}_{2}(r)-u_{2}(r)\right) r d r\right) \\
& +2 \pi\left(\int_{0}^{r_{*}}\left(\hat{f}_{2}(r)-f_{1}(r)\right) r d r\right)=V .
\end{aligned}
$$

Введем в рассмотрение функционал, включающий в себя поверхностную энергию и энергию силы тяжести. Поверхностная энергия, в свою очередь, состоит из части, соответствующей свободной поверхности жидкого моста, и части, соответствующей контакту жидкости с твердым телом. Исследуемый функционал можно записать в виде

$$
\begin{aligned}
& J\left\{u_{1}(r), u_{2}(r)\right\}=2 \pi \int_{r_{*}}^{r_{1}}\left\{\alpha_{34} \sqrt{1+\left(u_{1}^{\prime}\right)^{2}}\right. \\
& +\frac{1}{2} g \rho\left(u_{1}-f_{1}\right)^{2} \\
& \left.+\left(\alpha_{13}-\alpha_{14}\right) \sqrt{1+\left(f_{1}^{\prime}\right)^{2}}\right\} r d r+2 \pi \int_{r_{*}}^{r_{2}}\left\{\alpha_{34} \sqrt{1+\left(u_{2}^{\prime}\right)^{2}}\right. \\
& \left.-\frac{1}{2} g \rho\left(\hat{f}_{2}-u_{2}\right)^{2}+\left(\alpha_{23}-\alpha_{24}\right) \sqrt{1+\left(f_{2}^{\prime}\right)^{2}}\right\} r d r \\
& +2 \pi \int_{0}^{r_{*}}\left\{\left(\alpha_{13}-\alpha_{14}\right) \sqrt{1+\left(f_{1}^{\prime}\right)^{2}}+\left(\alpha_{23}-\alpha_{24}\right)\right. \\
& \left.\times \sqrt{1+\left(f_{2}^{\prime}\right)^{2}}+\frac{1}{2} g \rho\left(\hat{f}_{2}-f_{1}\right)^{2}\right\} r d r
\end{aligned}
$$

где $g$ - ускорение силы тяжести, $\rho-$ плотность жидкости. Таким образом, получили изопериметрическую задачу: найти минимум функционала (2) при условии, что функционал (1) принимает заданное значение $V$. В соответствии с теоремой Эйлера об изопериметрических задачах вводим расширенный функционал

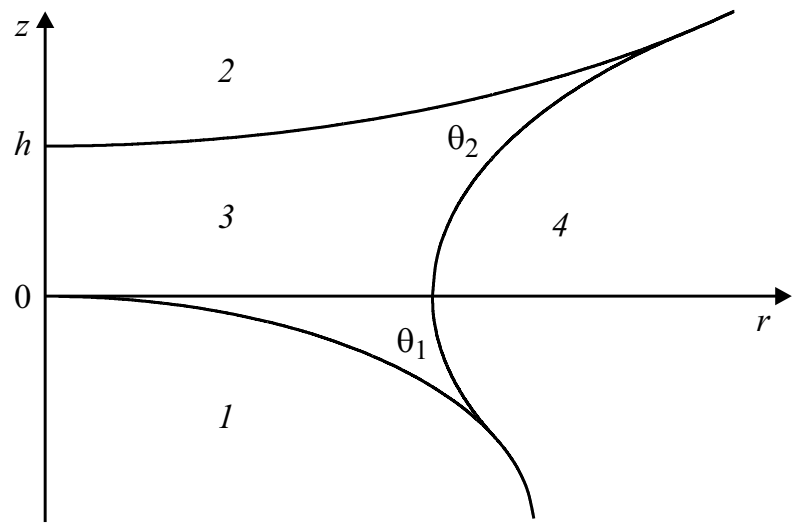

Рис. 1. Вертикальный осесимметричный жидкий мост между двумя твердыми выпуклыми поверхностями. 1 и 2 - твердые среды, 3 - жидкая среда, 4 - газообразная; $\theta_{1}, \theta_{2}-$ углы смачивания.

$(\lambda-$ множитель Лагранжа):

$$
\begin{aligned}
& J\left\{u_{1}(r), u_{2}(r)\right\}+\lambda I\left\{u_{1}(r), u_{2}(r)\right\} \\
& =2 \pi \int_{r_{*}}^{r_{1}} H_{1}\left(r, u_{1}(r), u_{1}^{\prime}(r)\right) d r \\
& +2 \pi \int_{r_{*}}^{r_{2}} H_{2}\left(r, u_{2}(r), u_{2}^{\prime}(r)\right) d r \\
& +2 \pi \int_{0}^{r_{*}} H_{3}(r) d r,
\end{aligned}
$$

где

$$
\begin{gathered}
H_{1}=\left\{\alpha_{34} \sqrt{1+\left(u_{1}^{\prime}\right)^{2}}+\frac{1}{2} g \rho\left(f_{1}-u_{1}\right)^{2}\right. \\
\left.+\left(\alpha_{13}-\alpha_{14}\right) \sqrt{1+\left(f_{1}^{\prime}\right)^{2}}+\lambda\left(u_{1}-f_{1}\right)\right\} r, \\
H_{2}=\left\{\alpha_{34} \sqrt{1+\left(u_{2}^{\prime}\right)^{2}}-\frac{1}{2} g \rho\left(\hat{f}_{2}-u_{2}\right)^{2}\right. \\
\left.+\left(\alpha_{23}-\alpha_{24}\right) \sqrt{1+\left(f_{2}^{\prime}\right)^{2}}+\lambda\left(\hat{f}_{2}-u_{2}\right)\right\} r, \\
H_{3}=\left\{\frac{1}{2} g \rho\left(\hat{f}_{2}-f_{1}\right)^{2}+\left(\alpha_{13}-\alpha_{14}\right) \sqrt{1+\left(f_{1}^{\prime}\right)^{2}}\right. \\
\left.+\left(\alpha_{23}-\alpha_{24}\right) \sqrt{1+\left(f_{2}^{\prime}\right)^{2}}+\lambda\left(\hat{f}_{2}-f_{1}\right)\right\} r .
\end{gathered}
$$

Выполнив варьирование расширенного функционала, получим два уравнения Эйлера и два условия трансверсальности. Введем безразмерные переменные $\xi=r / V^{1 / 3}$, $w_{i}(\xi)=u_{i}(r) / V^{1 / 3}, \quad i=1,2, \varphi_{1}(\xi)=f_{1}(r) / V^{1 / 3}, \quad \hat{\varphi}_{2}(\xi)=$ $=\hat{h} / V^{1 / 3}+f_{2}(r) / V^{1 / 3}=h+\varphi_{2}(\xi)$ и безразмерные параметры $\mu=\lambda V^{1 / 3} / \alpha_{34}, B=g \rho V^{2 / 3} / \alpha_{34}$. Безразмерная постоянная $B$ - число Бонда. 
В безразмерном виде задача примет следующий вид: уравнения Эйлера для нижней и верхней ветви соответственно

$$
\begin{aligned}
& \frac{d}{d \xi}\left(\frac{\xi w_{1}^{\prime}(\xi)}{\sqrt{1+\left(w_{1}^{\prime}(\xi)\right)^{2}}}\right)=B \xi\left(w_{1}(\xi)-\varphi_{1}(\xi)\right)+\mu \xi \\
& \xi_{*}<\xi<\xi_{1} \\
& \frac{d}{d \xi}\left(\frac{\xi w_{2}^{\prime}(\xi)}{\sqrt{1+\left(w_{2}^{\prime}(\xi)\right)^{2}}}\right)=B \xi\left(\hat{\varphi}_{2}(\xi)-w_{2}(\xi)\right)-\mu \xi \\
& \xi_{*}<\xi<\xi_{2}
\end{aligned}
$$

условия трансверсальности

$$
\begin{aligned}
& \frac{1+\varphi_{1}^{\prime}\left(\xi_{1}\right) w_{1}^{\prime}\left(\xi_{1}\right)}{\sqrt{1+\left(w_{1}^{\prime}\left(\xi_{1}\right)\right)^{2}}}=\alpha_{10} \sqrt{1+\left(\varphi_{1}^{\prime}\left(\xi_{1}\right)\right)^{2}}, \\
& \alpha_{10}=\frac{\alpha_{14}-\alpha_{13}}{\alpha_{34}}, \\
& \frac{1+\varphi_{2}^{\prime}\left(\xi_{2}\right) w_{2}^{\prime}\left(\xi_{2}\right)}{\sqrt{1+\left(w_{2}^{\prime}\left(\xi_{2}\right)\right)^{2}}}=\alpha_{20} \sqrt{1+\left(\varphi_{2}^{\prime}\left(\xi_{2}\right)\right)^{2}}, \\
& \alpha_{20}=\frac{\alpha_{24}-\alpha_{23}}{\alpha_{34}}
\end{aligned}
$$

условия соприкосновения моста с дном и крышкой

$$
\begin{gathered}
w_{1}\left(\xi_{1}\right)=\varphi_{1}\left(\xi_{1}\right), \\
w_{2}\left(\xi_{2}\right)=h+\varphi_{2}\left(\xi_{2}\right) ;
\end{gathered}
$$

условие непрерывности профиля моста в „шейке“

$$
w_{1}\left(\xi_{*}\right)=w_{2}\left(\xi_{*}\right)
$$

условия вертикальности касательной в „шейке“

$$
w_{1}^{\prime}\left(\xi_{*}\right)=-\infty, w_{2}^{\prime}\left(\xi_{*}\right)=+\infty ;
$$

условие сохранения объема

$$
\begin{aligned}
& 2 \pi\left(\int_{\xi_{*}}^{\xi_{1}}\left(w_{1}(\xi)-\varphi_{1}(\xi)\right) \xi d \xi+\int_{\xi_{*}}^{\xi_{2}}\left(\hat{\varphi}_{2}(\xi)-w_{2}(\xi)\right) \xi d \xi\right) \\
& \quad+2 \pi\left(\int_{0}^{\xi_{*}}\left(\hat{\varphi}_{2}(\xi)-\varphi_{1}(\xi)\right) \xi d \xi\right)=1
\end{aligned}
$$

\section{2. Алгоритм решения задачи}

Вначале найдем углы смачивания $\theta_{1}, \theta_{2}$ (углы между касательными к кривым, определяющим заданные поверхности, и касательными к профилю боковой поверхности жидкого моста в точках $\xi_{1}, \xi_{2}$ соответственно). Выражения для косинусов этих углов имеют вид

$$
\begin{aligned}
& \cos \left(\theta_{i}\right)=\cos \left(\arctan \left(w_{i}^{\prime}\left(\xi_{i}\right)\right)-\arctan \left(\varphi_{i}^{\prime}\left(\xi_{i}\right)\right)\right) \\
& =\frac{1+w_{i}^{\prime}\left(\xi_{i}\right) \varphi_{i}^{\prime}\left(\xi_{i}\right)}{\sqrt{1+\left(w_{i}^{\prime}\left(\xi_{i}\right)\right)^{2}} \sqrt{1+\left(\varphi_{i}^{\prime}\left(\xi_{i}\right)\right)^{2}}}, \quad i=1,2 .
\end{aligned}
$$

Следовательно, условия трансверсальности (6), (7) дают $\cos \left(\theta_{i}\right)=\alpha_{i 0}, i=1,2$, т. е. условия Дюпре-Юнга [9] (формула (1.4)). Видно, что в случае, когда заданные поверхности представляют собой параллельные плоскости, приходим к выражениям, приведенным в [1].

Для построения эффективного алгоритма решения задачи (4)-(12) выполним нормирование на величину $\xi_{*}$ следующим образом:

новая независимая переменная $\eta=\xi / \xi_{*}$;

новые искомые функции $v_{i}(\eta)=w_{i}(\xi) / \xi_{*}, i=1,2$; модифицированные заданные функции $\psi_{1}(\eta)=\varphi_{1}(\xi) / \xi_{*}$, $\hat{\psi}_{2}(\eta)=h / \xi_{*}+\varphi_{2}(\xi) / \xi_{*}=H+\psi_{2}(\eta)$.

Введем обозначения: $b=B\left(\xi_{*}\right)^{2}-$ модифицированное число Бонда; $M=\mu \xi_{*}-$ модифицированный множитель Лагранжа.

В новых переменных уравнения (4) и (5) примут вид

$$
\begin{aligned}
& \frac{d}{d \eta}\left(\frac{\eta v_{1}^{\prime}(\eta)}{\sqrt{1+\left(v_{1}^{\prime}(\eta)\right)^{2}}}\right)=b\left(v_{1}(\eta)-\psi_{1}(\eta)\right) \eta+M \eta, \\
& 1<\eta<\eta_{1}, \\
& \frac{d}{d \eta}\left(\frac{\eta v_{2}^{\prime}(\eta)}{\sqrt{1+\left(v_{2}^{\prime}(\eta)\right)^{2}}}\right)=b\left(\hat{\psi}_{2}(\eta)-v_{2}(\eta)\right) \eta-M \eta, \\
& 1<\eta<\eta_{2} .
\end{aligned}
$$

Интегрируя эти уравнения, получаем

$$
\begin{aligned}
& \frac{\eta v_{1}^{\prime}(\eta)}{\sqrt{1+\left(v_{1}^{\prime}(\eta)\right)^{2}}}=b \int_{1}^{\eta}\left(v_{1}(s)-\psi_{1}(s)\right) s d s \\
& +\frac{M\left(\eta^{2}-1\right)}{2}+C_{1}, \\
& \frac{\eta v_{2}^{\prime}(\eta)}{\sqrt{1+\left(v_{2}^{\prime}(\eta)\right)^{2}}}=b \int_{1}^{\eta}\left(\hat{\psi}_{2}(s)-v_{2}(s)\right) s d s \\
& -\frac{M\left(\eta^{2}-1\right)}{2}+C_{2} .
\end{aligned}
$$

Переходя в (15) и (16) к пределу при $\eta \rightarrow 1$ с учетом условий (11), находим постоянные интегрирования $C_{1}$ и $C_{2}: C_{1}=-1, C_{2}=1$. Разделив обе части $(15),(16)$ на $\eta$, приводим эти уравнения к виду

$$
\begin{aligned}
& \frac{v_{1}^{\prime}(\eta)}{\sqrt{1+\left(v_{1}^{\prime}(\eta)\right)^{2}}}=\frac{1}{\eta}\left[b \int_{1}^{\eta}\left(v_{1}(s)-\psi_{1}(s)\right) s d s\right. \\
& \left.+\frac{M\left(\eta^{2}-1\right)}{2}-1\right] \equiv-\Phi_{1}(\eta), \\
& \frac{v_{2}^{\prime}(\eta)}{\sqrt{1+\left(v_{2}^{\prime}(\eta)\right)^{2}}}=\frac{1}{\eta}\left[b \int_{1}^{\eta}\left(\hat{\psi}_{2}(s)-v_{2}(s)\right) s d s\right. \\
& \left.-\frac{M\left(\eta^{2}-1\right)}{2}+1\right] \equiv \Phi_{2}(\eta) .
\end{aligned}
$$


Введенные вспомогательные функции $\Phi_{1}(\eta)$ и $\Phi_{2}(\eta)$ должны удовлетворять неравенствам $0<\Phi_{1}(\eta) \leq 1$, $\eta \in\left[1, \eta_{1}\right], \quad 0<\Phi_{2}(\eta) \leq 1, \quad \eta \in\left[1, \eta_{2}\right]$ для обеспечения нужных знаков функциям $v_{1}^{\prime}(\eta)$ и $v_{2}^{\prime}(\eta)$ (см. выше). Разрешая (17) и (18) относительно производных, приходим к следующим уравнениям:

$$
\begin{aligned}
& \frac{d v_{1}}{d \eta}=-\frac{\Phi_{1}(\eta)}{\sqrt{1-\left(\Phi_{1}(\eta)\right)^{2}}} \\
& \frac{d v_{2}}{d \eta}=\frac{\Phi_{2}(\eta)}{\sqrt{1-\left(\Phi_{2}(\eta)\right)^{2}}}
\end{aligned}
$$

Из уравнений (19) следует

$$
\left.\frac{d v_{1}}{d \eta}\right|_{\eta=1}=-\infty,\left.\frac{d v_{2}}{d \eta}\right|_{\eta=1}=+\infty
$$

т. е. выполнение условий (11) вертикальности касательной в шейке. В окрестности точки $\eta=1$ функции $\Phi_{1}(\eta)$ и $\Phi_{2}(\eta)$ представляются в виде $\Phi_{i}(\eta)=1+O(\eta-1)$, $i=1,2$, поэтому особенности в правых частях (19) являются интегрируемыми, и можно получить выражения для искомых функций $v_{1}(\eta)$ и $v_{2}(\eta)$

$$
\begin{array}{r}
v_{1}(\eta)=\int_{\eta}^{\eta_{1}} \frac{\Phi_{1}(s) d s}{\sqrt{1-\left(\Phi_{1}(s)\right)^{2}}}+\psi_{1}\left(\eta_{1}\right), \\
v_{2}(\eta)=H+\psi_{2}\left(\eta_{2}\right)-\int_{\eta}^{\eta_{2}} \frac{\Phi_{2}(s) d s}{\sqrt{1-\left(\Phi_{2}(s)\right)^{2}}},
\end{array}
$$

при этом выполнены аналоги условий соприкосновения моста с дном и крышкой: $v_{1}\left(\eta_{1}\right)=\psi_{1}\left(\eta_{1}\right)$, $v_{2}\left(\eta_{2}\right)=H+\psi_{2}\left(\eta_{2}\right)$. Выполняя аналог условия (10), приходим к соотношению

$$
\begin{aligned}
& H=\psi_{1}\left(\eta_{1}\right)-\psi_{2}\left(\eta_{2}\right)+\int_{1}^{\eta_{1}} \frac{\Phi_{1}(s) d s}{\sqrt{1-\left(\Phi_{1}(s)\right)^{2}}} \\
& +\int_{1}^{\eta_{2}} \frac{\Phi_{2}(s) d s}{\sqrt{1-\left(\Phi_{2}(s)\right)^{2}}} .
\end{aligned}
$$

Тогда выражение для функции $v_{2}(\eta)$ примет вид

$$
v_{2}(\eta)=\psi_{1}\left(\eta_{1}\right)+\int_{1}^{\eta_{1}} \frac{\Phi_{1}(s) d s}{\sqrt{1-\left(\Phi_{1}(s)\right)^{2}}}+\int_{1}^{\eta} \frac{\Phi_{2}(s) d s}{\sqrt{1-\left(\Phi_{2}(s)\right)^{2}}} .
$$

Условия трансверсальности (6) и (7), используя соотношения (19), можно переписать в виде

$$
\begin{aligned}
& \sqrt{1-\left(\Phi_{i}\left(\eta_{i}\right)\right)^{2}}+(-1)^{i} \psi_{i}^{\prime}\left(\eta_{i}\right) \Phi_{i}\left(\eta_{i}\right) \\
& =\alpha_{i 0} \sqrt{1+\left(\psi_{i}^{\prime}\left(\eta_{i}\right)\right)^{2}}, \quad i=1,2
\end{aligned}
$$

Разрешая эти уравнения относительно величин $\Phi_{1}\left(\eta_{1}\right)$ и $\left.\Phi_{2}\left(\eta_{2}\right)\right)$, получаем

$$
\begin{aligned}
& \Phi_{i}\left(\eta_{i}\right)=\alpha_{i}\left(\eta_{i}\right), \quad \alpha_{i}\left(\eta_{i}\right) \\
& =\frac{\sqrt{1-\left(\alpha_{i 0}\right)^{2}}+(-1)^{i} \alpha_{i 0} \psi_{i}^{\prime}\left(\eta_{i}\right)}{\sqrt{1+\left(\psi_{i}^{\prime}\left(\eta_{i}\right)\right)^{2}}}, i=1,2 .
\end{aligned}
$$

Соотношения (24) после подстановки в них выражений для $\Phi_{1}\left(\eta_{1}\right)$ и $\Phi_{2}\left(\eta_{2}\right)$ дают уравнения для нахождения величин $\eta_{1}$ и $\eta_{2}$ :

$$
\begin{aligned}
& \frac{1}{2} M \eta_{1}^{2}+\alpha_{1}\left(\eta_{1}\right) \eta_{1}-\left[1+\frac{1}{2} M-b\right. \\
& \left.\times \int_{1}^{\eta_{1}}\left(v_{1}(s)-\psi_{1}(s)\right) s d s\right]=0 \\
& \frac{1}{2} M \eta_{2}^{2}+\alpha_{2}\left(\eta_{2}\right) \eta_{2}-\left[1+\frac{1}{2} M\right. \\
& \left.+b \int_{1}^{\eta_{2}}\left(\hat{\psi}_{2}(s)-v_{2}(s)\right) s d s\right]=0 .
\end{aligned}
$$

Перепишем в новых переменных условие сохранения объема (12):

$$
\begin{aligned}
& \xi_{*}^{3}\left(\int _ { 1 } ^ { \eta _ { 1 } } \left(v_{1}(s)-\psi_{1}(s) s d s+\int_{1}^{\eta_{2}}\left(\hat{\psi}_{2}(s)-v_{2}(s)\right) s d s\right.\right. \\
& \left.+\int_{0}^{1}\left(\hat{\psi}_{2}(s)-\psi_{1}(s)\right) s d s\right)=\frac{1}{2 \pi} .
\end{aligned}
$$

Интегрируя по частям, приведем соотношение (27) к виду

$$
\begin{aligned}
& \xi_{*}=\left\{\pi \left[\int_{1}^{\eta_{1}} \frac{\Phi_{1}(s) s^{2} d s}{\sqrt{1-\left(\Phi_{1}(s)\right)^{2}}}+\int_{1}^{\eta_{2}} \frac{\Phi_{2}(s) s^{2} d s}{\sqrt{1-\left(\Phi_{2}(s)\right)^{2}}}\right.\right. \\
& \left.\left.+\int_{0}^{\eta_{1}} \psi_{1}^{\prime}(s) s^{2} d s-\int_{0}^{\eta_{2}} \psi_{2}^{\prime}(s) s^{2} d s\right]\right\}^{-1 / 3} .
\end{aligned}
$$

Таким образом, получены все соотношения, необходимые для решения задачи.

Пусть число Бонда - малый параметр рассматриваемой задачи. Предлагаем следующий алгоритм ее решения для заданных значений $\alpha_{10}, \alpha_{20}, \hat{h}$.

Организуем итерационный процесс по малому параметру $b$ (главный итерационный процесс).

\section{1. Первая итерация (построение нулевого приближения)}

На первой итерации считаем $b=0$ (строим приближенное решение задачи без учета действия силы 
тяжести). Задаем значение параметра $M$ из диапазона допустимых значений и определяем функции $\Phi_{1}(\eta)$, $\Phi_{2}(\eta)$ по формулам (17), (18). Допустимыми значениями параметра $M$ будем считать те, при которых каждое из уравнений (25), (26) имеет по крайней мере один положительный корень больший единицы. Для положительных допустимых значений существует только один такой корень у каждого из уравнений, а для отрицательных допустимых значений - по два таких корня у каждого из уравнений. В последнем случае одному значению параметра $M$ будут соответствовать четыре решения задачи, которые определяются выбором корней уравнений (25), (26). Варианты выбора корней: $(++)$ - максимальные корни этих уравнений; вариант $(+-)-$ максимальный корень уравнения $(25)$ и минимальный (26); вариант $(-+)-$ наоборот, минимальный (25) и максимальный (26) и, наконец, вариант (- -) - оба корня минимальные.

Далее решаем нелинейные уравнения (25),(26) относительно величин $\eta_{1}, \eta_{2}$. Для этого сводим решение нелинейных уравнений к решению последовательности квадратных уравнений методом итераций (вспомогательный итерационный процесс, необходимый только при построении нулевого приближения, чтобы запустить главный итерационный процесс и учесть форму заданных поверхностей, между которыми находится жидкий мост). А именно полагаем $f_{1}^{\prime}(r)=0, f_{2}^{\prime}(r)=0$, тогда $\alpha_{i}\left(\eta_{i}\right)=\sqrt{1-\left(\alpha_{i 0}\right)^{2}}, i=1,2$ (жидкий мост между плоскостями и уравнения (25), (26) - квадратные), находим положительные корни квадратных уравнений большие единицы. Находим величину $\xi_{*}$ по формуле (28). Используем эти найденные значения корней $\eta_{1}, \eta_{2}$ для вычисления $\alpha_{i}\left(\eta_{i}\right), i=1,2$. Снова решаем квадратные уравнения $(25),(26)$ и находим $\xi_{*}$ по формуле (28). Продолжаем этот процесс до тех пор, пока разность значений корней на двух последовательных шагах вспомогательного итерационного процесса не станет меньше заданного значения, определяющего точность вычислений для нулевого приближения. Находим величину $H$ по формуле (22), функции $v_{1}(\eta), v_{2}(\eta)$ по формулам (20), (23) и, наконец, $\xi_{1}, \xi_{2}, h, w_{1}(\xi), w_{2}(\xi), \theta_{1}, \theta_{2}$. На этом процесс построения нулевого приближения для заданных значений $\alpha_{i 0}, i=1,2$ и выбранного значения параметра $M$ заканчивается. Одному значению параметра $M$ могут соответствовать до четырех различных профилей.

Если мы хотим ограничиться нахождением нулевого приближения, то перебираем все допустимые значения параметра $M$, строим зависимость высоты жидкого моста от параметра $M$ и находим решение, соответствующее заданному значению высоты.

\section{2. Вторая и последующие итерации главного итерационного процесса}

Если же мы не ограничиваемся рассмотрением жидкого моста в невесомости, то продолжаем процесс вычислений для того же выбранного значения параметpa $M$. А именно находим значение параметра $b=B\left(\xi_{*}\right)^{2}$, определяем новые вспомогательные функции $\Phi_{1}(\eta)$, $\Phi_{2}(\eta)$ по формулам $(17),(18)$, используя для вычислений функции $v_{1}, v_{2}, \psi_{1}, \psi_{2}$ с первой итерации. Уравнения $(25),(26)$ на второй итерации представляют собой квадратные уравнения относительно $\eta_{1}$ и $\eta_{2}$ с постоянными коэффициентами, поскольку при вычислении этих коэффициентов мы используем величины, полученные на предыдущей итерации. Находим $\eta_{1}, \eta_{2}, \xi_{*}, H, v_{1}(\eta)$, $v_{2}(\eta)$ и $\xi_{1}, \xi_{2}, h, w_{1}(\xi), w_{2}(\xi)$, далее находим новое значение параметра $b=B\left(\xi_{*}\right)^{2}$ и делаем третью итерацию аналогичным образом. И так далее, пока отличия в значениях корней $\eta_{1}, \eta_{2}$ на двух последовательных шагах главного итерационного процесса не станут меньше заданной величины, определяющей точность решения задачи.

Теперь выполняем все описанные вычисления для каждого из допустимых значений параметра $M$, строим зависимость высоты жидкого моста $h$ от параметра $M$, и находим решение задачи, соответствующее заданному значению высоты моста. Максимальное число таких решений равно четырем.

\section{3. Пример расчета жидкого моста между сферами}

Применим приведенный выше алгоритм для расчета частного случая - вертикального катеноидального жидкого моста между сферами. Пусть дно - сфера радиуса $R_{1}=6: \varphi_{1}(\xi)=-R_{1}+\sqrt{R_{1}^{2}-\xi^{2}} ;$ крышка - сфера радиуса $R_{2}=8: \hat{\varphi}_{2}(\xi)=h+\phi_{2}(\xi), \phi_{2}(\xi)=R_{2}-\sqrt{R_{2}^{2}-\xi^{2}}$. Расчеты проводим для числа Бонда, равного 0.25 , $\alpha_{10}=0.5, \alpha_{20}=0.7$, и выполняем первую и вторую итерации. Приводим результаты расчетов после первого

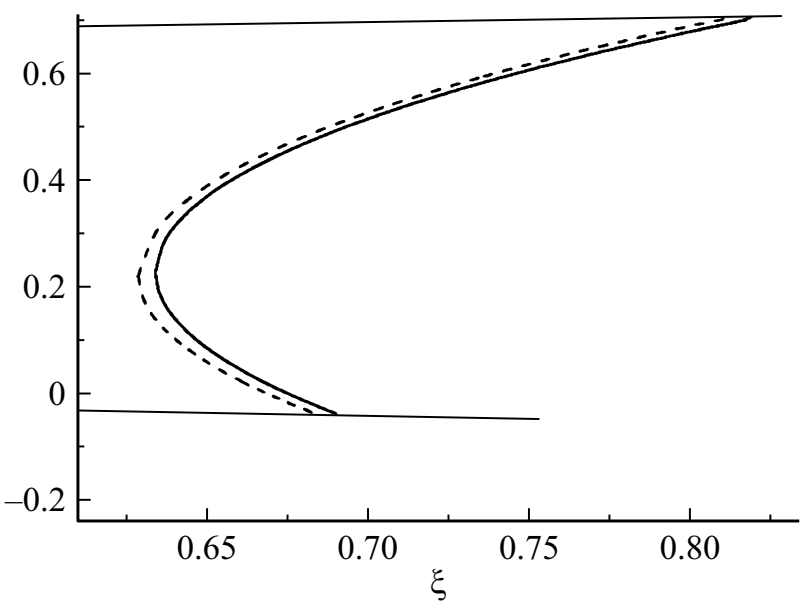

Рис. 2. Профили жидкого моста после первого шага главного итерационного процесса (сплошная линия) и после второго шага (штриховая линия). Случай $\alpha_{1}=0.5, \alpha_{2}=0.7$, $M=-0.01, R_{1}=6, R_{2}=8, B=0.25, b=0.1$. Вариант $(++)$. 
шага главного итерационного процесса, так как отличия в решениях малы и графики профилей практически сливаются. Рис. 2 иллюстрирует эту ситуацию. Выбран

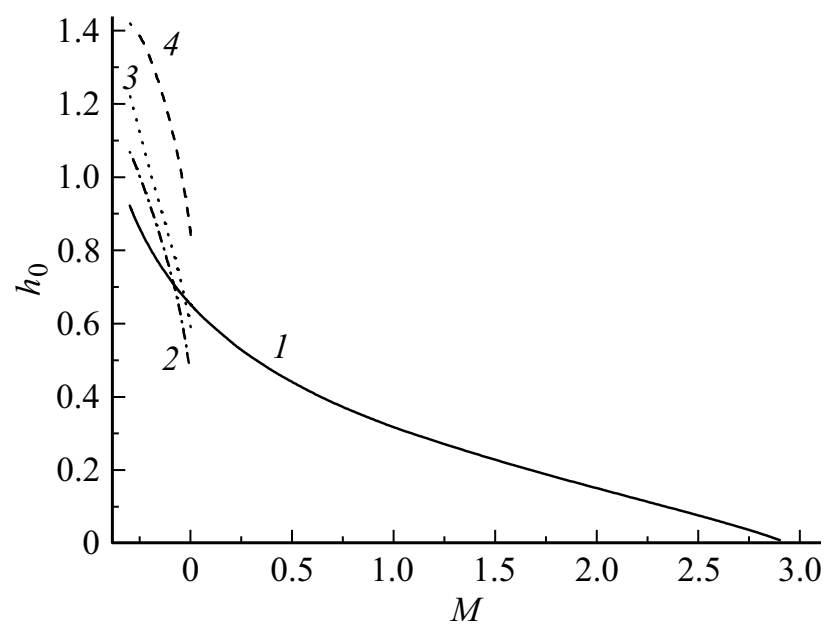

Рис. 3. Зависимость высоты жидкого моста $h_{0}$ от параметра $M$. График 1 соответствует варианту $(++)$ выбора значений корней; график $2-$ варианту $(+-)$; график $3-(-+)$; графика $4-(--)$.
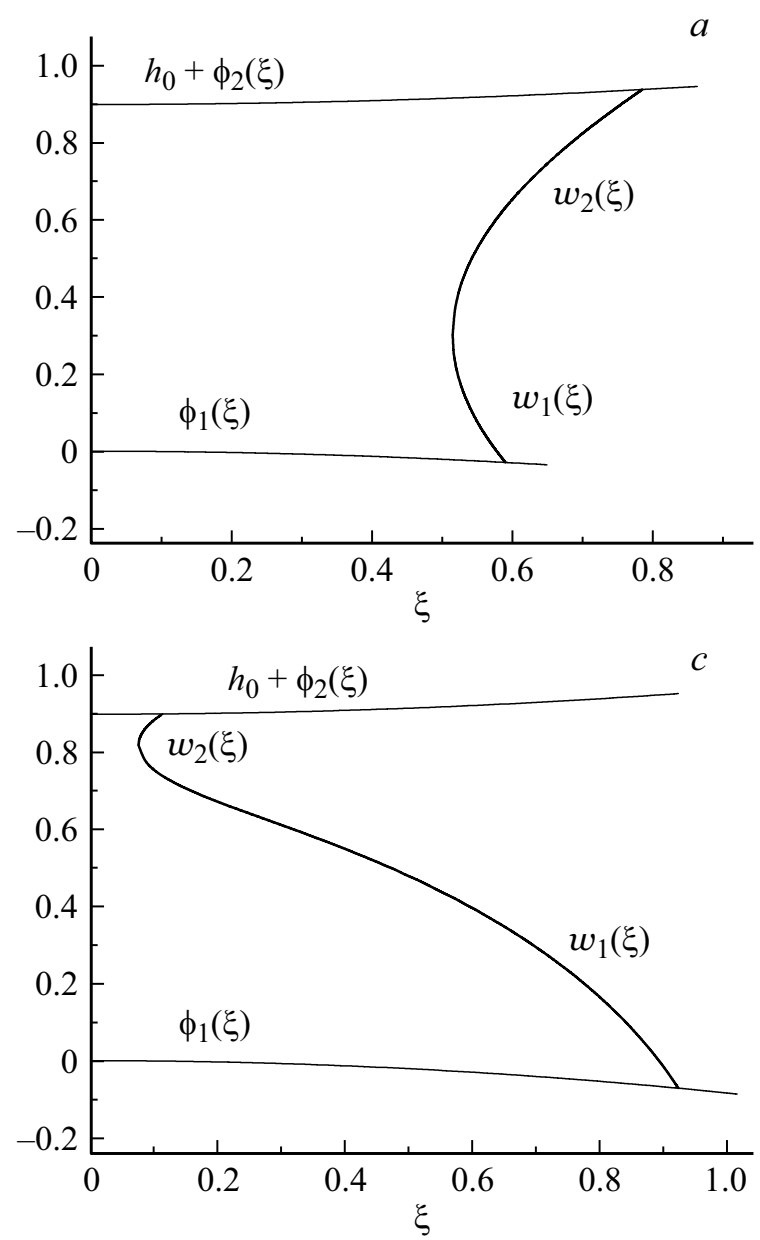

вариант $(++)$, поскольку значения параметра $b$, характеризующего радиус шейки в этом варианте, максимальны.

Видна хорошая сходимость главного итерационного процесса. Что касается сходимости вспомогательного итерационного процесса, то четыре его шага обеспечивают точность $0.5 \%$. Введем обозначение $h_{0}=H \xi_{*}$, где значения $H$ и $\xi_{*}$ берутся после четырех шагов.

На рис. 3 приведен график зависимости высоты моста $h_{0}$ (величины, определяющей расстояние между сферами) от параметра $M$. Для допустимых положительных значений параметра $M$ имеем одну кривую, а для допустимых отрицательных значений $M$ получаем четыре различные кривые, соответствующие четырем вариантам выбора корней уравнений $(25),(26)$. Существует максимальное значение высоты жидкого моста (для значений высоты превышающих это значение решений нет). Есть интервалы изменения высоты, соответствующие наличию одного, двух, трех и четырех решений. Кроме того, из этого рисунка видно, что в отличие от случая жидкого моста между двумя параллельными твердыми плоскостями, здесь существует конечное положительное значение параметра $M$, соответствующее точке касания сфер.
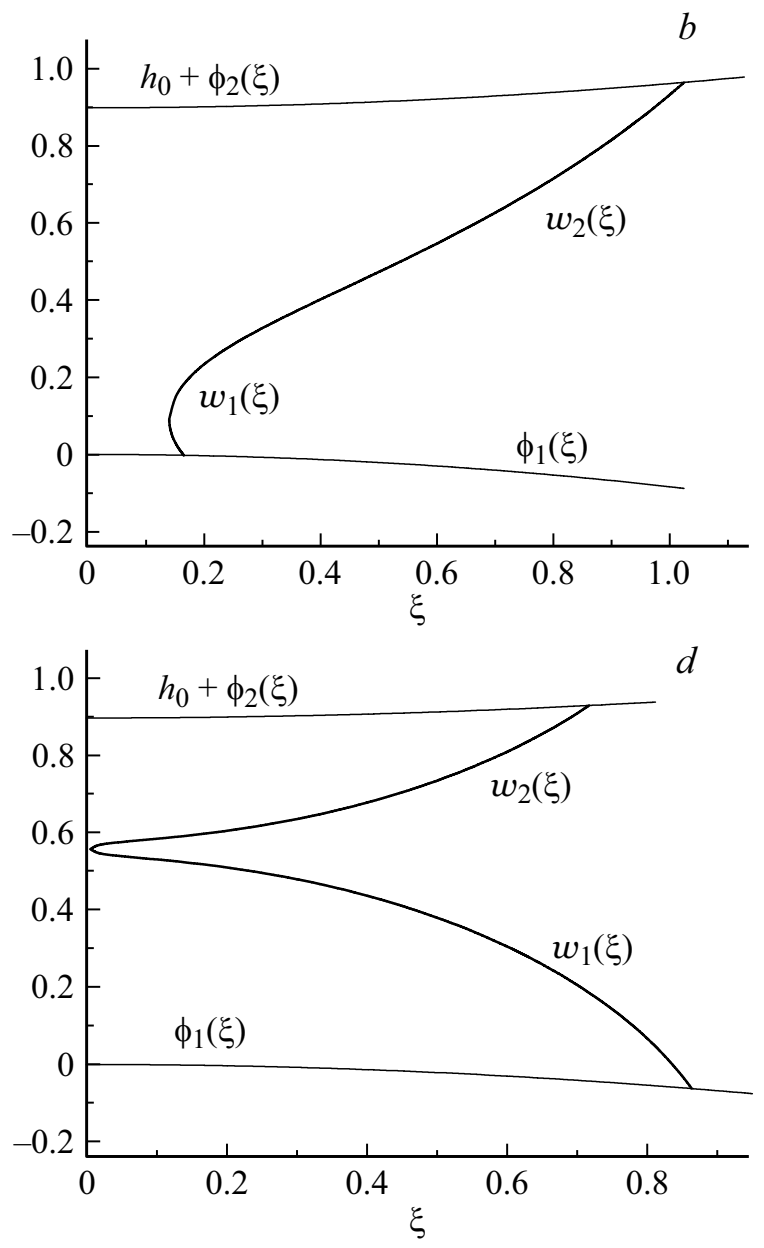

Рис. 4. Четыре различных профиля жидкого моста, соответствующие одному значению его высоты. $a-$ вариант $(++)$, $M=-0.284 ; b-(+-), M=-0.185 ; c-(-+), M=-0.14 ; d-(--), M=-0.013$. 
И, наконец, на рис. 4 приведены четыре различных профиля боковой поверхности жидкого моста между сферами, соответствующие одному значению высоты моста $h_{0}=0.9$ и четырем различным значениям параметра $M$. Заметим, что график $(a)$, соответствующий варианту $(++)$, согласуется с фотографиями, полученными в ходе эксперимента и приведенными в работе [6], в то время как остальные три графика отражают другие теоретически возможные профили.

\section{Заключение}

Предложен эффективный алгоритм решения задачи нахождения формы боковой поверхности вертикального катеноидального жидкого моста между твердыми выпуклыми поверхностями малой кривизны с учетом силы тяжести. Дана вариационная постановка задачи. Решение находится методом итераций в предположении малости числа Бонда, причем старт берется от случая жидкого моста между параллельными плоскостями. Обнаружено отсутствие единственности решения задачи. Установлено, что максимальное число различных решений для фиксированного набора заданных параметров равно четырем. В качестве примера построено приближенное решение задачи о форме жидкого моста между сферами. Исследована зависимость числа решений от высоты моста. Приведены четыре различных профиля боковой поверхности моста, соответствующие одному значению его высоты.

\section{Конфликт интересов}

Авторы заявляют, что у них нет конфликта интересов.

\section{Список литературы}

[1] Е.В. Галактионов, Н.Е. Галактионова, Е.А. Тропп. ЖТФ, 87 (10), 1475 (2017). DOI: 10.21883/jtf.2017.10.44989.2297n [E.V. Galaktionov, N.E. Galaktionova, E.A. Tropp. Tech. Phys., 62 (10), 1482 (2017). DOI: 10.1134/S1063784217100115]

[2] E.V. Galaktionov, N.E. Galaktionova, E.A. Tropp. J. Phys.: Conf. Ser., 1400 (4), 044025(7) (2019). DOI: $10.1088 / 1742-6596 / 1400 / 4 / 044025$

[3] П.И. Антонов, Л.М. Затуловский, А.С. Костыгов, Д.И. Левинзон, С.П. Никаноров, В.В. Пеллер, В.А. Татарченко, В.С. Юферев. Получение профилированных монокристаллов и изделий способом Степанова. Под ред. В.Р. Регеля, С.П. Никанорова. (Наука, Л., 1981), 280 с.

[4] M. Haynes, S.B.G. O’Brien, E.S. Benilov. Phys. Fluids., 28, 042107(17) (2016). DOI: 10.1063/1.4946001

[5] G. Gagneux, O. Millet. Transp. Porous Med., 105, 117 (2014). DOI: $10.1007 / \mathrm{s} 11242-014-0363-y$

[6] H.N.G. Nguyen, O. Millet, G. Gagneux. Continuum Mech. Thermodyn., 31, 225 (2019). DOI: 10.1007/s00161-018-0658-2

[7] P.I.C. Teixeira, M.A.C. Teixeira. J. Phys.: Condens. Matter., 32 (3), 034002(13) (2020). DOI: 10.1088/1361-648X/ab48b7
[8] E. Bendito, M.J. Bowick, A. Medina. J. Geom. Symmetry Phys., 33, 27 (2014). DOI: 10.7546/jgsp-33-2014-27-45

[9] В.Г. Бабский, Н.Д. Копачевский, А.Д. Мышкис. Гидромеханика невесомости. Под ред. А.Д. Мышкиса. (Наука, М., 1976), $504 \mathrm{c}$. 